\title{
COSEISMIC AND POSEISMIC GRAVITY CHANGES OBTAINED FROM GRACE SATELLITE DATA DURING THE POWERFUL TOHOKU-OKI EARTHQUAKE OF 11 MARCH 2011
}

\author{
M.Akhoondzadeh $^{\text {a, }}$, M.A.Sharifi ${ }^{\text {b }}$, M.Shahrisvand ${ }^{\text {a }}$ * \\ ${ }^{a}$ Remote sensing division, Department of Surveying and Geomatic Engineering, College of Engineering, \\ University of Tehran, Iran-(m.shahrisvand)@ut.ac.ir \\ ${ }^{\mathrm{b}}$ Geodesy division, , Department of Surveying and Geomatic Engineering, College of Engineering, \\ University of Tehran, Iran
}

KEY WORDS: Gravity, Earthquake, GRACE

\begin{abstract}
:
In this paper, we study the gravitational gradient changes induced 11 March $2011 \mathrm{Mw} 9.0$ Tohoku-Oki earthquake using data gathered by Gravity Recovery and Climate Experiment (GRACE) satellite. Previous studies have demonstrated the capability of GRACE data for observing the coseismic gravity change concerning the Tohoku earthquake. However, we show for the first time that changes of the coseismic and postseismic gravitational gradient components are detectable without doing any post-processing and filtering. It can be seen that the derivative of gravitational potential amplify the high-frequency components of the earth gravity field. Therefore, gravitational gradient changes delineate more clearly the rupture lines and reveal refined mass redistribution features caused by the studied earthquake.
\end{abstract}

\section{INTRODUCTION}

Earthquake is one of the most devastating disasters that human beings have to face. Every year thousands of humans are killed by earthquake. If we can know more about the earthquake mechanism, we can better protect ourselves from earthquake. There are many different prospects of doing research on earthquake and the final goal all the researches want to achieve is the prediction of the earthquake. In earthquake studies, the Earth gravity field method can efficiently detect the signals in underground of land and oceans in comparing to the other geodetic methods. The gravity change signal demonstrated underground processes of the earthquakes that can help the scientists to more deeply complementation of earthquake mechanism. The gravitational potential changes due to earthquakes are attributable to three factors (Okubo 1991, Okubo 1992) (1) Density perturbation $-\rho \nabla \cdot u$ of the half space material, where $\rho$ is the original of unperturbed half space and $u$ is displacement field in half-space (2) The surface mass density change $\rho \Delta h$ owing to the uplift /subsidence $\Delta h$ of the originally flat half-space surface, and (3) Attraction of mass with density $\rho^{\prime}$ filling into the cavity created by tensile fracturing.

Variation in the Earth's gravity field due earthquakes have been formulated nearly two decades ago (Okubo 1991, Sun and Okubo 1993). The first reliable detection of gravity change was made by an array of superconducting gravimeters after the 2003 Tokachi - Oki earthquake $(\mathrm{Mw}=8.0)$, Japan (Imanishi, Sato et al. 2004).

\footnotetext{
* Corresponding author
}

The Tohoku-Oki earthquake, Mw 9.0, which occurred at 05:46 UT, 11 March, 2011, at the Japan Trench east of NE Japan, ruptured the fault as large as $500 \mathrm{~km} \bullet \sim 200 \mathrm{~km}$ (Ozawa, Nishimura et al. 2011). Earthquake of this size and magnitude cause mass-dislocation on the scale that is measurable by Gravity Recovery and Climate Experiment (GRACE) gravity satellite mission. Displacement taking place immediately during the earthquake are called coseismic. The dislocated masses disturb the isotactic equilibrium of the crust and mantle, thus including a long term post-seismic relaxation process.

The GRACE mission is a spaceborne gravity mission launched in March 2002 jointly by the National Aeronautics and Space Administration (NASA) and the German Aerospace Center (DLR) (Tapley, Bettadpur et al. 2004). GRACE is a twinsatellite formation, with two identical spacecrafts chasing each other in similar near-polar orbits. A K-band ranging system provides the biased inter-satellite range as well as its derivatives with respect to time. In addition, both satellites are equipped with geodetic GPS receivers as well as accelerometers for removing non-gravitational forces prior data analysis. The GRACE satellites have the capability to accurately measure temporal variations in the Earth's gravitational field and can thus be used to estimate large-scale mass redistributions within the Earth system, e.g. terrestrial hydrological water balance, ocean mass variations, sea-level rise and glacier ablations. Earthquake cause mass redistribution in the Earth's crust and upper mantle, and permanently disturb Earth's gravity field in free space, which would be detected by GRACE measurements. The two-dimensional distribution of coseismic gravity changes have been recovered for the first time by Level-1 GRACE range and range rate measurements after the great Sumatra-Andaman (SA) earthquake $(\mathrm{Mw}=9.1), 2004$ December 26 (Han, Shum et 
al. 2006). Ogawa and Heki (2007) processed the GRACE monthly gravitational field data for the first time to drive postseismic signal due the Sumatra earthquake. Chen et al. (2007) showed that improved GRACE monthly gravity field from the University of Texas Center for Space Research (CSR) are able to reveal the coseismic change with greater spatial resolution. Other studies using GRACE to detect or constrain coseismic or post-seismic deformations of great earthquake include: i) Panet, Mikhailov et al. 2007, Han, Sauber et al. 2008, Han and Simons 2008, de Linage, Rivera et al. 2009, Einarsson, Hoechner et al. 2010, Broerse, Vermeersen et al. 2011, Cambiotti, Bordoni et al. 2011, Wang, Shum et al. 2012, Han, Riva et al. 2013 for the 2004 Sumatra-Andaman and Nias earthquake; ii) Han, Sauber et al. 2010, Heki and Matsuo 2010, Wang, Shum et al. 2012, Han, Riva et al. 2013 for the 2010 Chile Maule event and iii) Han 2011, Matsuo and Heki 2011, Wang, Shum et al. 2012, Han, Riva et al. 2013 for the 2011 Tohoku-Oki earthquake.

Previous studies have demonstrated the capability of GRACE data for observing the coseismic gravity changes concerning the Tohoku earthquake. In this study, we show that some of components gravitational gradient changes due to the coseismic and postseismic deformations are detectable in both spatial and temporal domain without any performing of post-processing and filtering for the Tohoku earthquake.

\section{GRACE DATA PROCESSING}

We used CSR (RL) 05 GRACE Level 2 (L2) monthly gravitational field data product, which are composed of fully normalized SH coefficients complete to degree and order 60 , corresponding to a spatial resolution $333 \mathrm{~km}$ or longer. A total 116 monthly gravitational field solution covering from 2003 January to 2013 February are used. We replaced the Earth's oblateness values $\left(\mathrm{C}_{20}\right)$ with those from Satellite Laser Ranging because of their poor accuracy. The $\mathrm{SH}$ coefficients $\mathrm{C}_{\mathrm{nm}}, \mathrm{S}_{\mathrm{nm}}$ of each monthly field are then used to compute monthly full gravitational gradients tensor (second derivative of the potential) in spherical coordinate. Then our attention is given local mass redistribution the local north-earth-down frame at the point with spherical coordinates $(r, \varphi, \lambda)$ is introduced: $\mathrm{x}$ axis is directed to the north, the $\mathrm{y}$-axis to the east, and the $\mathrm{z}$-axis downward. According to the principal of curvilinear coordinate, the full gravitational gradient tensor is this local NED frame is obtained.

A problem that all users of monthly GRACE gravity field solutions face is the presence of correlated and wavelengthdependent noise in the provided spherical harmonic coefficients. This manifests itself in north-south directed, elongated features in the derived gravity models, usually described as striping patterns. Usually de-striping and spatial smoothing filters applied for reduce impact of high-frequency components of GRACE SH coefficients and estimated of timevariable signals. However we did not apply any de-striping and spatial smoothing procedure in order to preserving original signals as much as possible.

To suppress seasonal variations and isolate coseismic change, we compute the difference between two mean gravity field after and before the earthquake. The mean gravity filed before earthquake is compute from 24 solutions, February 2009 to February 2011, and the mean gravity filed after earthquake is computed from the last 21 solutions, March 2011 to February 2013. There are three gaps between 2011 March to 2013
February. Since a large part of the deformation is in the ocean, therefore the hydrological model (e.g. GLDAS) cannot be used to remove seasonal variations.

This difference include post-seismic signals associated TohokuOki earthquake. To remove the mentioned effects in the GRACE data, we adopt at a $1^{\circ} \times 1^{\circ}$ grid a time-dependent function (de Linage, Rivera et al. 2009):

$$
y(t)=a+b\left(1-e^{-t / \tau}\right)
$$

Where $a$ is constant term, $\tau$ and $\mathrm{b}$ are the relaxation time and total post-seismic gravitational gradient change at end of the relaxation. Then the coseismic gravitational gradient changes were extracted by computing the difference between two mean gravity fields before and after earthquake.

To analyze coseismic and post-seismic deformation in the time domain, the time series of gravitational gradient changes from 2003 to 2013 have been computed. In order to improve the illustration of the rupture feature and postseismic effects, we removed seasonal variations (annual and semiannual and S2 tidal wave) from time series using least squares analysis. The March 2011 solution is excluded in the least squares fit.

\section{RESULTS AND DISCUSSION}

Unfortunately there are few observations for great undersea earthquakes, since traditional geodetic measurements, such as GPS and InSAR, can only measure the deformation of land surface and have poor sensitivity to occurrence slip far offshore. Physical quantities are measured by GRACE mission, such as geoidal height, gravitational acceleration and its gradient changes, which represent the total surface and subsurface mass deformation by uniform coverage over both ocean and land, however, its application to earthquake studies is limited by its current relatively coarse spatial resolution. Thus, these quantities can be used to estimate the deformation associated with uplift/subsidence of the sea floor, which may have larger effect on disturbing the surrounding gravitational field than the visible land areas.

Large deformations caused by earthquakes are typically highly local, and large parts of the signal associated with the deformation, reside in the high frequency band. At the early phase of GRACE mission, the GRACE-detected mass redistributions were expressed using geoid height changes (Tapley, Bettadpur et al. 2004). As the accuracy of GRACE solutions were gradually improved, most studies use GRACEdetected 'surface mass change' or 'gravity change' to present their scientific results, since these quantities enhance the highfrequency components and hence reveal more detailed spatial features. In this study, we use GRACE-derived gravitational gradient changes to obtain finer signatures of certain geophysical phenomena. Wang et al. (2012a) shows the model predicted of vertical deformation (maximum degree of 60) due to Sumatra earthquake has more than 70 per cent of its energy residing in SH degrees higher than 35 . Therefore by amplifying the high frequency content, GRACE-derived gravitational gradient components help reveal more detailed information about the deformation that induced by an earthquake.

It is true that noises in the GRACE-derived high degree SH coefficients are amplified as well by driving the second derived of the potential changes. For small earthquakes, it is possible 
that noise be overcome of the signal, however for Tohoku-Oki earthquake coseismic gravity change is strong enough. In addition independence of the gravitational gradient tensor to GRACE stripy errors, cause increase signal to noise ratio.

We know that GRACE stripe errors are distributed in northsouth direction, thus these strips generate fluctuations in eastwest direction (Figure 1). Therefore by taking derivative to the $\mathrm{x}$-axis (north direction) these variations dramatically suppress. Figure 2 shows $\Delta \mathrm{V}_{\mathrm{xz}}$ component change which related to Tohoku earthquake without applying any filters.

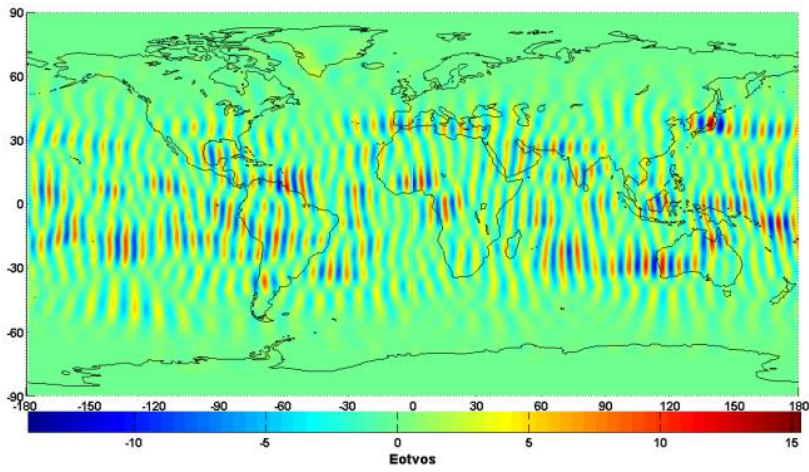

Figure 1. Eastern gravity gradient changes between the two mean gravity fields without filtering (in $\mathrm{E}$ unit).

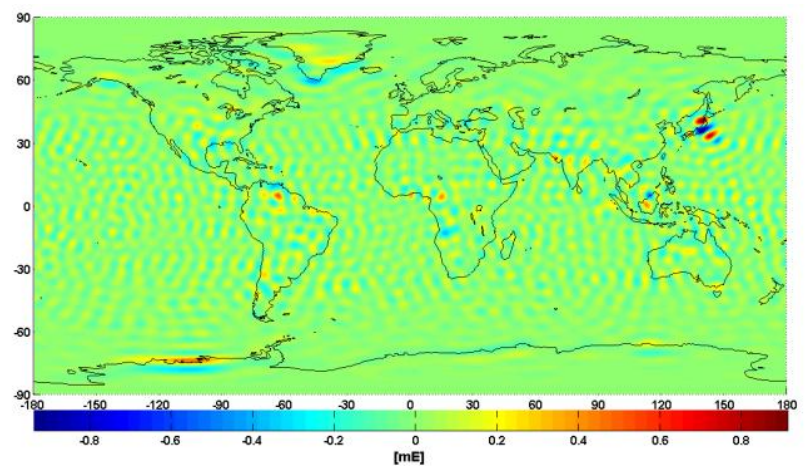

Figure 2. Northern gravity gradient changes between the two mean gravity fields without filtering (in unit of $\mathrm{mE}$ ).

However, we must note that horizontal derivative operator shifts the phase of the original anomaly distribution in spatial domain. By taking derivative in the $\mathrm{x}$-axis direction, we obtain gravitational signal variation in the $\mathrm{x}$-axis direction. As a result, peaks and troughs in $\Delta \mathrm{V}_{\mathrm{xz}}$ map indicate the edge of the anomaly source, not maximum mass anomaly. These peaks and troughs can be distinguished mass anomaly better than gravitational change, since peaks and troughs are sharpened due to enhancement of the high frequency components. Two peaks and two troughs can be found around the rupturing region in Figure 3c. By comparing $\Delta \mathrm{V}_{\mathrm{zz}}$ (Figure $3 \mathrm{f}$ ) with peaks and troughs of $\Delta \mathrm{V}_{\mathrm{xz}}$ component, we conclude that they indicate the location of the northern and southern mass anomaly, as a result of the large slip due to Tohoku-Oki earthquake. The zero contours in the $\Delta \mathrm{V}_{\mathrm{xz}}$ component show north-south edges. In Figure 2 features are related to non-seasonal hydrological effects (e.g. postglacial rebound in Northern Canada and ice-sheet melting in Greenland) dramatically suppressed. Since these features do not occur in spatial and temporal domain with high-frequency.
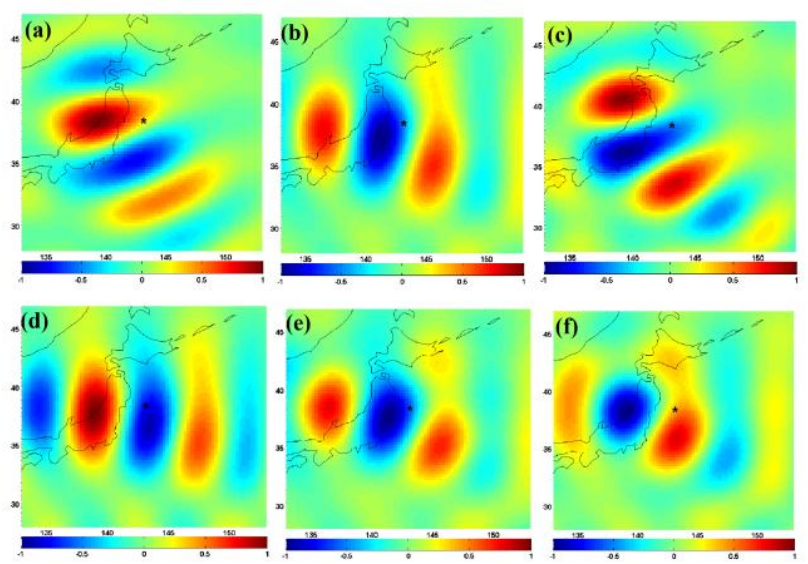

Figure 3. Gravitational gradient changes from GRACE data without applying any de-striping or spatial smoothing filter for component a) $\Delta \mathrm{V}_{\mathrm{xx}}$, b) $\Delta \mathrm{V}_{\mathrm{xy}}$, c) $\Delta \mathrm{V}_{\mathrm{xz}}$, d) $\Delta \mathrm{V}_{\mathrm{yy}}$, e) $\Delta \mathrm{V}_{\mathrm{yz}}$, and f) $\Delta \mathrm{V}_{\mathrm{zz}}$. The values have been normalized to better illustrate the changes. The black star shows the epicenter (38.1N, 142.9E).

Similar to the computed $\Delta \mathrm{V}_{\mathrm{xz}}$ and $\Delta \mathrm{V}_{\mathrm{xx}}$ components, $\Delta \mathrm{V}_{\mathrm{yz}}$ and $\Delta \mathrm{V}_{\mathrm{yy}}$ also show the location of edges anomaly in east-west direction. But by taking derivative to y-axis, GRACE stripy errors are enhanced. Therefore these components are dominated by noise without applying any de-striping and spatial smoothing filters (Figure 1).

As mentioned earlier $\Delta \mathrm{V}_{\mathrm{xz}}$ and $\Delta \mathrm{V}_{\mathrm{xx}}$ components dramatically suppress stripes errors. Traditionally, to obtained reasonable estimates from GRACE, various de-striping and spatial smoothing algorithm have to be applied to the original monthly gravitational field solution. These processes inevitably decrease of GRACE's spatial resolution, and decrease magnitude the true geophysical signals as well. In addition, in Tohoku earthquake with fault strike almost in the north-south direction, the bipolar gravity change signature will be partially interpreted as stripes by any de-striping filter and thus damped and distort to some extend due to the anisotropy of such filters.

To better analysis of the coseismic and post-seismic deformation, some points near of epicenter are selected and time series of the GRACE gravitational gradient change during the 10 year period are shown (Figure 4). There are significant jumps before and after earthquake in both uplift and seduction zones. After Tohoku-Oki earthquake, continued deformations in the same direction as coseismic deformation can be seen. Also different sing of gravitational gradient components changes in same location can be observed. The main reason is spatial horizontal phase shift in the mass anomaly, which produced by taking derivative in direction of $\mathrm{x}$-axis and $\mathrm{y}$-axis. In addition, in $\Delta \mathrm{V}_{\mathrm{xy}}$ time series is not seen any jump (Figure $4 \mathrm{~b}$ ), and it is probably due to the noise amplification by taking derivative in $y$ direction.

\section{CONCLUSIONS}

We show that monthly GRACE time variable gravity solutions, are able to monitor the gravitational gradient change signature of deformation due to the rupture from Tohoku-Oki earthquake $(\mathrm{Mw}=9.0)$ without applying any post-processing and filtering procedure. Traditionally, to obtained reasonable earthquake deformation signals from GRACE, various de-striping and spatial smoothing algorithms have to applied in order to reduce the high-frequency errors in the SH coefficients. Depending on 

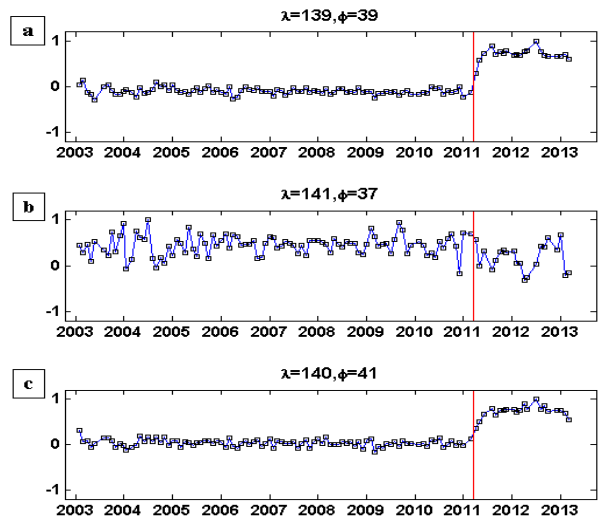
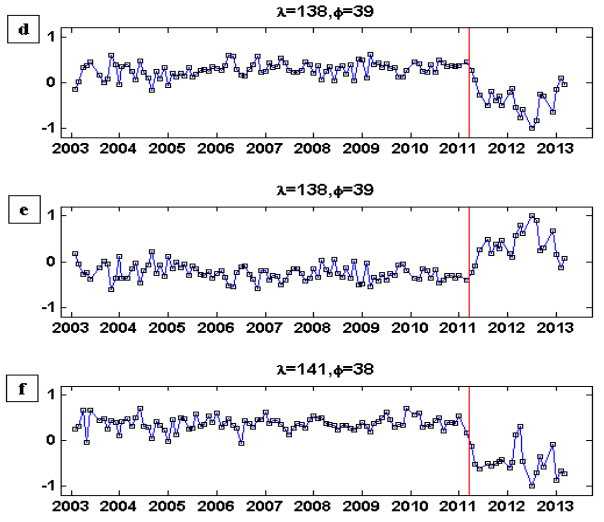

Figure 4. GRACE non-seasonal gravitational gradient change without applying any de-striping or spatial smoothing filter for component a) $\Delta \mathrm{V}_{\mathrm{xx}}$, b) $\Delta \mathrm{V}_{\mathrm{xy}}$, c) $\Delta \mathrm{V}_{\mathrm{xz}}$, d) $\Delta \mathrm{V}_{\mathrm{zz}}$, e) $\Delta \mathrm{V}_{\mathrm{yy}}$, and f) $\Delta \mathrm{V}_{\mathrm{yz}}$. Seasonal (annual and semiannual and $\mathrm{S} 2$ tidal wave) signals are removed from time series using least squares. The red line represents the time of Tohoku-Oki earthquake.

the type of the used post-processing algorithms, there could be significant trade-off between the attainable results of spatial resolution and accuracy of the GRACE estimated deformation. Independence of some gravitational gradient tensor to GRACE stripy errors can pay the way of estimation actual signal caused by earthquake without filtering. In addition for geophysical phenomena of regional scale which are dominated by high frequency content (such as earthquake), gravitational gradient changes are more useful. These components enhance high frequency components of the earth gravity field and reveal more detail in spatial and temporal domain.

\section{REFRENCES}

Broerse, D., et al. (2011). "Ocean contribution to co-seismic crustal deformation and geoid anomalies: application to the 2004 December 26 Sumatra-Andaman earthquake." Earth and Planetary Science Letters 305(3): 341-349.

Cambiotti, G., et al. (2011). "GRACE gravity data help constraining seismic models of the 2004 Sumatran earthquake." Journal of Geophysical Research 116(B10).

Chen, J. L., et al. (2007). "GRACE detects coseismic and postseismic deformation from the Sumatra-Andaman earthquake." Geophysical Research Letters 34(13).

de Linage, C., et al. (2009). "Separation of coseismic and postseismic gravity changes for the 2004 Sumatra-Andaman earthquake from $4.6 \mathrm{yr}$ of GRACE observations and modelling of the coseismic change by normal-modes summation." Geophysical Journal International 176(3): 695-714.

Einarsson, I., et al. (2010). "Gravity changes due to the Sumatra-Andaman and Nias earthquakes as detected by the GRACE satellites: a reexamination." Geophysical Journal International 183(2): 733-747.

Han, S.-C. (2011). "Contribution of satellite gravimetry to understanding seismic source processes of the 2011 TohokuOki earthquake." Geophysical Research Letters 38.

Han, S.-C., et al. (2013). "Source parameter inversion for recent great earthquakes from a decade-long observation of global gravity fields." Journal of Geophysical Research: Solid Earth 118(3): 1240-1267.

Han, S.-C., et al. (2010). "Regional gravity decrease after the 2010 Maule (Chile) earthquake indicates large-scale mass redistribution." Geophysical Research Letters 37(23).
Han, S.-C., et al. (2008). "Implications of postseismic gravity change following the great 2004 Sumatra-Andaman earthquake from the regional harmonic analysis of GRACE intersatellite tracking data." Journal of Geophysical Research 113(B11).

Han, S.-C. and F. J. Simons (2008). "Spatiospectral localization of global geopotential fields from the Gravity Recovery and Climate Experiment (GRACE) reveals the coseismic gravity change owing to the 2004 Sumatra-Andaman earthquake." Journal of Geophysical Research 113(B1).

Han, S. C., et al. (2006). "Crustal dilatation observed by GRACE after the 2004 Sumatra-Andaman earthquake." Science 313(5787): 658-662.

Heki, K. and K. Matsuo (2010). "Coseismic gravity changes of the 2010 earthquake in central Chile from satellite gravimetry." Geophysical Research Letters 37(24).

Imanishi, Y., et al. (2004). "A network of superconducting gravimeters detects submicrogal coseismic gravity changes." Science 306(5695): 476-478.

Matsuo, K. and K. Heki (2011). "Coseismic gravity changes of the 2011 Tohoku-Oki earthquake from satellite gravimetry." Geophysical Research Letters 38(7).

Ogawa, R. and K. Heki (2007). "Slow postseismic recovery of geoid depression formed by the 2004 Sumatra-Andaman Earthquake by mantle water diffusion." Geophysical Research Letters 34(6).

Okubo, S. (1991). "Potential and gravity changes raised by point dislocations." Geophysical Journal International 105(3): 573-586.

Okubo, S. (1992). "Gravity and potential changes due to shear and tensile faults in a half-space." Journal of Geophysical Research 97(B5): 7137-7144.

Ozawa, S., et al. (2011). "Coseismic and postseismic slip of the 2011 magnitude-9 Tohoku-Oki earthquake." Nature 475(7356): 373-376.

Panet, I., et al. (2007). "Coseismic and post-seismic signatures of the Sumatra 2004 December and 2005 March earthquakes in GRACE satellite gravity." Geophysical Journal International 171(1): 177-190.

Sun, W. and S. Okubo (1993). "Surface potential and gravity changes due to internal dislocations in a spherical earth-I. Theory for a point dislocation." Geophysical Journal International 114(3): 569-592. 
Tapley, B. D., et al. (2004). "GRACE measurements of mass variability in the Earth system." Science 305(5683): 503-505.

Wang, L., et al. (2012a). "Gravitational gradient changes following the 2004 December 26 Sumatra-Andaman Earthquake inferred from GRACE." Geophysical Journal International: nono.

Wang, L., et al. (2012b). "Coseismic and postseismic deformation of the 2011 Tohoku-Oki earthquake constrained by GRACE gravimetry." Geophysical Research Letters 39(7).

Wang, L., et al. (2012c). "Coseismic slip of the $2010 \mathrm{Mw} 8.8$ Great Maule, Chile, earthquake quantified by the inversion of GRACE observations." Earth and Planetary Science Letters 335-336: 167-179. 